\title{
Des poèmes... et ils sont partis
}

La fiction dans les récits d'exploration au Tibet

\section{Samuel Thévoz}

\section{(2) OpenEdition \\ Journals}

Édition électronique

URL : http://journals.openedition.org/lhomme/22922

DOI : 10.4000/lhomme.22922

ISSN : 1953-8103

Éditeur

Éditions de l'EHESS

\section{Édition imprimée}

Date de publication : 23 février 2012

Pagination : 7-30

ISSN : 0439-4216

\section{Référence électronique}

Samuel Thévoz, «Des poèmes... et ils sont partis », L'Homme [En ligne], 201 | 2012, mis en ligne le 15 février 2014, consulté le 01 mai 2019. URL : http://journals.openedition.org/lhomme/22922 ; DOI 10.4000//homme.22922

Ce document a été généré automatiquement le 1 mai 2019.

(c) École des hautes études en sciences sociales 


\title{
Des poèmes... et ils sont partis
}

\author{
La fiction dans les récits d'exploration au Tibet
}

\section{Samuel Thévoz}

UNE SIMPLE RECENSION bibliographique permet de prendre la mesure de l'essor, depuis les années 1980, des études historiennes et littéraires sur le récit de voyage ${ }^{1}$. La question du statut du récit de voyage comme genre factuel a longtemps entrainé une sorte de «division du travail» entre historiens de la littérature et du voyage, d'une part, et théoriciens du « fait littéraire », d'autre part. Du côté « historien », s'est développée une critique philologique méfiante vouée à la vérification des faits consignés dans les textes. Du côté « littéraire », la critique philologique a cédé le pas à la question de l'écriture et, partant, à l'analyse des éléments constitutifs du discours des voyageurs. D'une part, la critique s'est consacrée à une étude formaliste des topoï et isotopies propres au genre ${ }^{2}$ ou à une tradition plus spécifique ${ }^{3}$. D'autre part, pareille approche s'est prolongée sous l'égide de différents paradigmes en sciences humaines. Globalement, le fondement épistémologique de ces approches se rapporte à une conception « projective » du récit de voyage (Reichler 2002), verrouillant tour à tour ce dernier dans les déterminations d'une culture propre (c'est l'approche culturaliste, cf. Venayre 2002), dans les mécanismes profonds de la psyché (c'est l'approche psychanalytique et mythocritique, cf. Bishop 1989 ; Buisine 1998 ; Roudaut 1984), dans les stratégies impérialistes de domination de l'altérité (pour une approche postcoloniale, voir l'étude séminale de Said 1979) ou enfin dans le deuil du référent (l'« ostranénie » des formalistes russes acculturée non sans fascination par le structuralisme français) constitutif de toute mise en discours (pour une approche panfictionnaliste post-structuraliste, cf. Montalbetti 1997). Ces deux orientations critiques, réaliste et fictionnaliste (pour ne pas dire idéaliste), pour paraitre incommensurables, condamnent l'une comme l'autre le récit de voyage à une polarisation irréconciliable, laquelle en abolit du même coup la spécificité générique et les enjeux les plus fondamentaux.

2 Dans le présent article, je cherche à me dégager de ce qui a toutes les apparences d'un débat voué à l'aporie, dans la mesure où le couple véridictionnel vrai/faux recouvre, peu ou prou, de façon plus ou moins avouée dans les deux versants critiques évoqués, le binôme factuel/fiction. Aussi, sur un plan théorique, la notion de fiction est-elle 
radicalisée - elle devient de part et d'autre un synonyme de simulacre et de mensonge ${ }^{4}$. Le débat ne reposerait-il donc pas sur une insuffisance des définitions des genres et concepts pris à partie et sur une analyse trop rapide de leurs relations? À bien y regarder, est de ce fait entravée, sur le plan pratique de l'herméneutique textuelle, l'analyse au cas par cas des phénomènes d'ordre fictionnel et de leurs statuts cognitif et épistémique dans les récits des voyageurs. Ces questions ont fait l'objet des nombreuses recherches consacrées ces dernières années à la réhabilitation du concept de fiction. Toutefois, prenant un tour foncièrement théorique, la discussion me semble avoir perdu de vue l'hétérogénéité épistémologique et la variabilité phénoménale des manifestations fictionnelles dans les genres dits factuels, auxquels on rapporte généralement le récit de voyage. Il convient, à mon sens, de reprendre sur de nouvelles bases la question de la référentialité en prenant appui sur ce qui fait le fondement d'une définition du récit de voyage, à savoir son indexation sur l'expérience vécue du voyageur ${ }^{5}$.

Pour étayer ma réflexion et donner à voir une pluralité d'échanges possibles entre fiction et récit de voyage sans faire l'hypothèque de la valeur de connaissance assignable au genre, j'aimerais m'appuyer sur un corpus circonscrit de récits dont on verra qu'ils témoignent d'usages fort disparates de la fiction. Afin de donner une unité au parcours proposé ici, l'étude se concentrera sur la question du paysage dans les récits d'exploration au Tibet. Figurant parmi les enjeux majeurs du récit de voyage, le paysage a la particularité de pouvoir se rapporter à un topos, codé, du genre (la description de paysage comme lieu obligé du récit de choses vues), autant qu'à une expérience intime vécue in situ. Le texte d'un côté, le monde de l'autre. Ces deux polarités, loin d'écarteler le paysage entre des approches inconciliables, se verront reconfigurées dans l'analyse empirique, par le biais de la question de savoir ce que c'est que la fiction pour le voyageur qui raconte son voyage, quel statut il convient de lui attribuer. Ainsi, je chercherai moins à proposer ma théorie de la fiction qu'à montrer la redistribution nécessaire des termes du débat en fonction des usages qui en sont faits, des motivations qui se lisent dans les textes eux-mêmes.

\section{Du monde à la fiction : le topos du paysage tibétain}

4 Jouons, pour commencer, le jeu de l'approche qualifiée précédemment de «formaliste » en nous intéressant à un phénomène de circulation transgénérique, nous invitant à penser la question de la porosité des genres dits fictionnels et des genres dits factuels. En ce qui concerne la description du paysage tibétain, il est aisé d'identifier une modalité isotopique particulière dans des textes fictionnels, dans des textes scientifiques, dans des récits de voyage. Un tel type de description est à l'œuvre, par exemple, dans Robur-leConquérant de Jules Verne (2004 [1886]).

5 Le Tibet figure dans le roman comme l'un des passages obligés du tour du monde effectué par Robur à bord de son aéronef, L'Albatros. Contrairement à d'autres contrées traversées par les protagonistes, le Tibet ne sera que survolé et observé sous un angle panoramique. Dans cet extrait, l'on distingue clairement deux dimensions qui relèvent d'une littérarisation de la "matière » viatique et d'un imaginaire scientifique. La représentation du Tibet se fait par le biais, d'une part, d'une mise en texte très "rhétorique ", très travaillée et, d'autre part, d'une dimension propre au savoir viatique. Précisons ces deux manières de circonscrire le Tibet. La première se différencie par son caractère explicitement esthétique : 
«Le Tibet, - hauts plateaux sans végétation, de-ci de-là pics neigeux, ravins desséchés, torrents alimentés par les glaciers, bas-fonds avec d'éclatantes couches de sel, lacs encadrés dans des forêts verdoyantes. Sur le tout, un vent glacial »(ibid. : 102).

«Magnifique disposition de ce chaos de montagnes! Partout des sommets blancs. Pas de lacs, mais des glaciers qui descendent jusqu'à dix mille pieds de la base. Plus d'herbe, rien que de rares phanérogames sur la limite de la vie végétale. Plus de ces admirables pins et cèdres, qui se groupent en forêts splendides aux flancs inférieurs de la chaîne. Plus de ces gigantesques fougères ni de ces interminables parasites, tendus d'un tronc à l'autre, comme dans les sous-bois de la jungle. Aucun animal, ni chevaux sauvages, ni yaks, ni bœufs tibétains. Parfois une gazelle égarée jusque dans ces hauteurs. Pas d'oiseaux, si ce n'est quelques couples de ces corneilles qui s'élèvent jusqu'aux dernières couches de l'air respirable »(ibid. : 106).

Dans cet extrait, la description est d'ordre négatif, soustractif et thématise de manière très dense l'impossibilité à représenter le paysage tibétain. Elle se marque ainsi par une structure syntaxique nominale et par une répétition de tournures privatives («plus de... rien que... », " pas de... mais... ", " ni... ni... », "sans ", " aucun »); par un lexique se rapportant aux thèmes de la stérilité et de l'inhumain (par certains substantifs : «la neige », « la glace », « le sel »; et certains adjectifs : « desséchés », « blancs », " rares »), ainsi que de la démesure (" hauts ", «bas-fonds ", « limite », " sommets », " jusque » [à deux reprises], "s'élèvent », " dernières couches »). Sur un mode proche de la théologie négative, la connaissance est ici d'ordre apophatique : la seule connaissance possible est l'absence même du connaissable, et cette connaissance se rapporte à une jouissance esthétique ("magnifique disposition de ce chaos de montagnes!» $»^{6}$ ). En termes de généalogie, il est possible de rapporter ce motif aux descriptions des missionnaires jésuites du XVII ${ }^{\mathrm{e}}$ siècle ${ }^{7}$ et, plus près de Verne, à la manière dont un orientaliste comme Théodore Pavie dresse un bilan des connaissances sur le Tibet dans un célèbre article de la Revue des Deux Mondes en $1847^{8}$.

Dans le même passage, la seconde manière de circonscrire le Tibet se distingue par son caractère épistémique et positif. Les lignes qui précèdent encadrent cette seconde modalité descriptive :

« En réalité, trois chaînes coupent successivement la route de l'Inde, quand on vient du nord. Les deux septentrionales, entre lesquelles s'était glissé l'Albatros, comme un navire entre d'énormes écueils, sont les premiers degrés de l'Asie centrale. Ce furent d'abord le Kouen-Loun, puis le Karakoroum, qui dessinent cette vallée longitudinale et parallèle à l'Himalaya, presque à la ligne de faîte où se partagent les bassins de l'Indus, à l'ouest, et du Brahmapoutre, à l'est.

Quel superbe système orographique! Plus de deux cents sommets déjà mesurés, dont dix-sept dépassent vingt-cinq mille pieds! Devant l'Albatros, à huit mille huit cent quarante mètres, s'élevait le mont Everest. Sur la droite le Dwalaghiri, haut de huit mille deux cents. Sur la gauche, le Kinchanjunga, haut de huit mille cinq cent quatrevingt douze, relégué au deuxième rang depuis les dernières mesures de l'Everest »(Jules Verne 2004 [1886] : 104).

8 Par une transition brutale, la locution adverbiale «En réalité » substitue à l'expérience esthétique du chaos la beauté de l'ordre... "Quel superbe système orographique!» s'exclame le narrateur comme par symétrie aux paragraphes précédents. La description se démarque alors par une saturation de chiffres $^{9}$, de relevés orographiques, de résultats scientifiques. La juxtaposition de ces deux modalités descriptives constitue un topos de la description du Tibet. On pourrait aller jusqu'à dire que Verne, dans son roman, construit et formalise une catégorie de représentation du Tibet. Par ailleurs, la fiction romanesque, 
comprise dans un contexte qui est celui du $\mathrm{XIX}^{\mathrm{e}}$ siècle, participe d'une logique générale, d'un imaginaire social (Laboulais-Lesage 2004, Venayre 2002 et 2006), où savoirs et littérature s'interpénètrent. Mais par son attention esthétique, le roman de Verne se démarque comme œuvre de fiction et renforce la dimension symbolique de la représentation.

\section{De la fiction au monde : défamiliarisation et bénéfice cognitif de la fiction}

Distinguer ici une dimension scientifique d'une dimension plus littéraire et esthétique comme polarités d'un modèle isotopique de représentation du Tibet invite donc à penser fiction romanesque et récit de voyage au sein d'une histoire des savoirs et des représentations. Issu de récits de voyage et de textes scientifiques, ce modèle s'accomplit par le roman puis est reversé dans l'imaginaire social du voyage, ainsi offert à l'interprétation et aux appropriations des lecteurs de la Troisième République et, bien entendu, des voyageurs, au Tibet en particulier ${ }^{10}$. Peut-on attester que les explorateurs se réapproprient de tels motifs fictionnels? Si tel est le cas, la thèse " projective » s'imposet-elle de droit?

Pour se convaincre de la circulation de la fiction dans le récit de voyage, lisons comment un explorateur comme Fernand Grenard, dont le voyage au Tibet est de peu postérieur à la publication de Robur, fait usage de la fiction.

11 En effet, on retrouve dans son récit du voyage, Le Tibet. Le pays et les habitants (1904) ${ }^{11}$, une combinaison stylistique et thématique qui nous rappelle la description vernienne: modalité apophatique de la description et savoir viatique positif. Notons dans les lignes qui suivent, lesquelles ouvrent pour ainsi dire le récit de la traversée du Tibet, la séquence reconduisant de manière topique la première modalité (apophatique) :

«Sa vallée est absolument aride et déserte; pas une touffe d'herbe, pas une trace

d'animal, pas un vol d'oiseau, rien qu'un peu d'eau qui court »(Grenard 1904 : 41).

12 Notons ensuite, en lien avec la seconde modalité (savoir viatique positif), les nombreuses notations de temps, d'altitudes, de distances et, dans l'ensemble, une description détaillée et attentive à la dimension biophysique du paysage :

«Le 27, nous arrivâmes au bord et non loin de la source de la branche la plus importante et la plus méridionale du Kara mouren. Les origines de cette rivière étaient dès lors complètement reconnues. Sa vallée, haute de 5200 mètres, large de trois kilomètres, au fond uni comme un plancher, est, ainsi que les autres vallées de l'Arka tâgh, de nature schisteuse »(Ibid.).

13 Cette combinaison fondée sur la polarisation de la démesure par l'alternance des dimensions positives et négatives, mène, au terme de la description, à l'élaboration d'une représentation "picturale", une sorte d'épure quasi symbolique de la montagne se détachant des scories orographiques décrites précédemment :

«Près de nous, à notre gauche, se dressait une masse colossale de neige et de glace, puissamment établie sur sa vaste base, élançant à 7360 mètres son pic le plus élevé. C'est le point culminant non seulement de la chaîne, mais probablement aussi de toute la région entre le Turkestan et l'Himalaya. Longtemps il nous apparut, décroissant lentement à l'horizon derrière nous : à 160 kilomètres au sud nous apercevions distinctement encore sa pyramide de cristal, qui semblait trôner dans sa majesté immuable sur le peuple nombreux des monts »(Ibid.). 
Dans ce mouvement ultime, résumant une «palette» d'éléments et de formes primordiales, se dessine une icône du paysage tibétain qu'une tradition littéraire entretiendra durablement à travers le $\mathrm{xx}^{\mathrm{e}}$ siècle $^{12}$.

Afin de nuancer l'analyse des processus de circulation transgénérique, il est nécessaire de replacer le passage cité ci-dessus dans son contexte immédiat. En effet, il ponctue un long développement descriptif. L'explorateur, pour faire percevoir la spécificité du paysage tibétain, recourt précisément à la fiction sur un mode explicite :

"Les choses que nous avons vues au cours de cette longue marche sont des choses grandioses, sans doute, mais toujours les mêmes [...], des choses arides et tristes,

Deserts idle,

Rough quarries, rocks, and hills whose heads touch heaven,

d'immenses pays où rien ne passe que le vent, où rien ne passe que des phénomènes géologiques. Si le vaillant Maure n'avait eu de plus intéressants sujets d'entretien, il aurait eu peu de chance de gagner le cœur le mieux disposé. Je ne saurais comme lui égayer et animer ces âpres paysages d'aventures merveilleuses, ni de personnages étranges qui ont la tête sous les épaules, ou le pied si vaste qu'ils peuvent s'en servir comme d'un parasol en le relevant sur leur tête. Durant soixante jours, l'homme ne se rappela à notre attention que par son absence, privant ainsi mes descriptions de tout autre élément de variété que nos propres peines et nos propres misères, sur lesquelles un explorateur aurait particulièrement mauvaise grâce à s'étendre et à s'attendrir, puisqu'il les affronte et les brave de son libre choix. Je serai donc bref »( Ibid. : 39-40).

La référence de la citation, même implicite, est facilement identifiable par la glose qui s'y rapporte. Comme ailleurs dans le récit, la citation témoigne de la culture lettrée du voyageur ${ }^{13}$; ici le drame shakespearien (Othello), plus loin, L'Enfer de Dante et, plus surprenant encore, un poème de Leconte de Lisle. Mais le traitement de la fiction doit retenir notre attention. Le commentaire est ici particulièrement développé et reprend tant l'énoncé (la topique de la montagne comme locus horribilis) que l'acte d'énonciation d'origine, ce qui permet au voyageur d'accentuer le fossé qui sépare la posture et la visée rhétorique du personnage shakespearien de sa propre situation, où l'horreur rhétorique des montagnes, pure formule poétique chez Shakespeare, s'incarne dans l'expérience vécue par l'explorateur. La manière dont est intégrée la citation, proche en cela du phénomène deproximisation décrit par Gérard Genette (Rabau 2002: 20), et la manière dont le voyageur s'approprie Shakespeare se caractérisent par la nécessité d'une suturation avec une œuvre reconnue comme non référentielle, induisant un « heurt » au niveau énonciatif, partiellement atténué par la paraphrase de la réplique d'Othello, par laquelle le voyageur rejoint son propos : définir la nature exploratrice de son voyage, nature à la fois liée à un imaginaire et à une situation bien réelle. Autrement dit, par son emploi de la citation, Fernand Grenard force à imaginer Othello racontant son voyage au Tibet, situation évidemment absurde... Par là, il fait usage d'un procédé littéraire qui a fait école dans les théories de la littérature, et dont Carlo Ginzburg (1989) a montré les propriétés cognitives : le procédé de la « défamiliarisation ». Ce jeu poétique s'opère dans une relation intertextuelle libre. S'insérant dans un contexte nouveau, la citation vise à un ajustement dans l'écriture, pour le lecteur, à une situation vécue dans le monde par le sujet. Notons encore que, parallèlement au réemploi citationnel, l'écriture viatique convoque ou congédie différents intertextes et différents «styles » discursifs. Ainsi, dans la suite du passage cité :

«De l'autre côté, nous campâmes parmi une confusion de blocs et de noires

lamelles schisteuses, sur le rebord d'une sorte d'entonnoir sombre, dominé par un 
chaos de montagnes aux figures bizarres qui semblaient comme étonnées de nous voir. La nuit, nous éprouvâmes un froid de trente degrés et perdîmes deux chevaux. C'était un beau début, un exorde ex abrupto d'une rude éloquence ; mais notre parti était pris, notre résolution fixée d'avance et nos oreilles fermées à tout argument contraire »(Grenard $1904: 40)$.

Dans le récit, l'emploi de motifs intertextuels (Othello) ${ }^{14}$, de figures rhétoriques (le monde comme chaos, le monde comme un livre où le voyage serait un parcours en «bibliothèque $»)^{15}$ et de représentations entérinées par le roman d'aventure par exemple (la solitude, le péril de l'inconnu, l'affrontement de la mort) fonctionne non comme voile sur le monde mais comme schème d'action. Dire ironiquement que les dangers encourus sont un "beau début, un exorde ex abrupto d'une rude éloquence ", outre présenter un jeu sur les mots particulièrement opportun, fournit au voyageur les moyens d'affronter et de se représenter son voyage non seulement comme celui d'un aventurier mais comme voyage d'exploration « pensable » et réalisable.

Quelles sont donc, après analyse, les spécificités de l'inscription d'un motif fictionnel dans le récit du voyage? Les observations proposées peuvent être regroupées en quatre modalités d'ouverture du texte sur ses contextes. D'abord, on évoquera une élaboration cognitive ; en amont du texte, on a ainsi observé comment un modèle de représentation permet de " saisir » l'insaisissable. Ensuite, on évoquera une dimension « éthique » : c'est la posture (Meizoz 2004) assumée par le narrateur de voyageur tour à tour explorateur et aventurier. On évoquera également une dimension pragmatique, en aval du texte; en faisant référence à une bibliothèque commune au voyageur et à ses lecteurs, l'auteur aménage les conditions de réception de son récit. Enfin, la présente description s'ouvre sur une dimension supplémentaire, que l'on devrait appeler épistémique, car elle concerne la connaissance vers laquelle s'oriente la description. La visée de la description paysagère qui, à proprement parler, enserre la citation s'ajuste à une dimension complètement étrangère à la réplique d'Othello, celle $d u$ «rien ne passe que des phénomènes géologiques ». Au-delà de la reconduction du topos tibétain, la description s'emploie à caractériser une " unité géographique ", notée comme débordant le point de vue restreint du voyageur et donnée par des « grilles » de savoir propres à la géographie de la fin du XIX siècle : les concepts de "région ", de "pays ", de "paysage ", d'" aspect ", de «caractère », de "système » soutiennent la description «littéraire » de Fernand Grenard. L'enjeu implicite de la description de l'«unité géographique » de l'espace traversé est ainsi relatif à la morphogenèse des systèmes montagneux. À cette période, les géologues commencent en effet à modéliser une proto-théorie de la tectonique des plaques. Épistémologiquement, la description de Grenard est donc à proprement parler d'ordre géologique: le voyageur cherche à comprendre la formation des montagnes et la structure tectonique dont elles sont l'épiphénomène, en comparant les "âges " géologiques dont témoignent l'aspect et la «nature» rocheuse du terrain livré à l'observation ${ }^{16}$. La synthèse qu'effectue la description à partir d'une analyse initiale n'est pas ici clôturée, mais donne à voir ses sutures. Ces sutures permettent au lecteur d'accéder à certaines étapes de la construction de la connaissance géographique, d'un mouvement qui passe par l'expérience vécue par le voyageur et la consignation de ses sensations, perceptions et observations. Ces lignes illustrent bien comment un paradigme épistémologique de type indiciaire (Ginzburg 1989) croise, d'une part, les dimensions analogiques de la description à valeur esthétique et, d'autre part, un paradigme de type galiéén qui constitue le pendant à l'observation géographique : la recherche de lois faisant système. 


\section{Voyager dans la fiction? Simulacres et évolution des représentations}

19 Au regard de l'approche intertextuelle, ces dimensions de la description de paysage dans le récit d'exploration, recouvrant des aspects cognitifs, éthiques, pragmatiques et épistémiques, étendent la notion de "bibliothèque» (Montalbetti 1997), qu'elle soit strictement littéraire ou extralittéraire, en ce sens qu'elle est aussi scientifique dans le récit d'exploration (Weber 2006). Mais la bibliothèque commune au voyageur et au lecteur est à elle seule insuffisante pour rendre compte de l'expérience viatique en ellemême. De surcroît, l'identification d'un répertoire emprunté à la fiction ne couvre guère l'étendue des processus d'échange entre fiction et expérience qui se manifestent dans le récit de voyage.

20 J'aimerais mettre en évidence pareil processus, toujours par le biais de la question du paysage, à partir d'un récit de 1912, Le Tibet révolté de Jacques Bacot. La complexité de la question s'illustre particulièrement dans ce récit dont il convient de mener une analyse pas à pas. Nous retrouvons plusieurs des modes «fictionnels» dégagés chez Verne et identifiés dans les passages précédents. Ainsi, le topos de la démesure :

"On arrive alors, dans des déserts glacés, si hauts qu'ils ne semblent plus appartenir à la terre, on escalade des montagnes affreuses, chaos d'abîmes noirs et de sommets blancs qui baignent dans le froid absolu du ciel »(Bacot $1912: 1$ ).

21 Ici, le modèle est en quelque sorte relégué au statut d'exergue, aux franges du récit; ce passage est le début de l'avant-propos et non une description de paysage au cours du voyage. Affiché d'entrée de jeu, ce modèle est de fait détaché de sa valeur descriptive opératoire. La "mise en scène» paratextuelle va à l'encontre de l'absorption intertextuelle (voir Rabau 2002 : 18); à bien y regarder, le modèle isotopique se propose ici comme un avant-propos au sens littéral. Le jeu intertextuel prend une dimension nouvelle, dans la mesure où s'opère une incorporation paradoxale des modèles de description connus du voyageur et du lecteur. L'avant-propos nous confie en quelque sorte : voici, avant de relater mon voyage, ce qui en a déjà été dit ; la lettre avant la lettre. En de multiples occurrences, le récit de Bacot développe ainsi une dimension réflexive où les représentations stéréotypées du Tibet, de ses paysages et de ses habitants, sont mises entre guillemets et prises en défaut (voir Thévoz 2007). Dans son premier récit, Bacot déclare d'emblée se méfier de la "belle confiance, de[s] bagages inutiles et de[s] tablettes » des explorateurs (1909: i-ii); plus loin, il souligne combien il est lui-même tributaire de représentations héritées, tout en exprimant l'ambivalence avec laquelle il envisage le paysage tibétain :

«J'éprouve alors avec intensité ce plaisir enfantin et rare qui est le faible et la force des voyageurs : être le premier homme à jouir de cette splendeur comme si elle m'attendait là depuis la création du monde »(ibid.: 84 ).

Enfin, dans son second récit, il bat en brèche les représentations du Tibet, confirmant ainsi le statut oblique à conférer à son avant-propos :

« Tout le Tibet n'est pas de glace et de désolation. Pourquoi fonder sa réputation sur ses parties inhabitées? Nous ne jugeons pas la Norvège par le Cap Nord »(Bacot 1912 : 93).

23 La description du paysage tibétain est un acte d'appropriation opéré par les explorateurs qui se dispensent ce faisant de porter quelque attention au regard des Tibétains eux- 
mêmes. Or, c'est précisément à une enquête sur la relation des Tibétains à leur « milieu ", pour reprendre un terme d'époque, que se livre Bacot, loin des préconceptions primitivistes et déterministes qui imprègnent la pensée ethnologique du tournant $d u \mathrm{xx}^{\mathrm{e}}$ siècle. De schèmes d'intelligibilité qu'ils étaient pour les prédécesseurs de Bacot, les modèles « littéraires » de description du Tibet autant que les critères «scientifiques » et positivistes, relégués au rang de "fictions", font maintenant écran, rendant imperceptible le Tibet. Selon Bacot, celui-ci aurait en effet été réduit à une pure fantasmagorie et une affabulation. En d'autres termes, les représentations héritées, mentionnées bien plus qu'employées, ne vont plus de soi. La fiction, qu'il faut comprendre ici dans le sens "antimimétique " de simulacre, devient l'objet d'une approche critique par le voyageur et se voit de facto disqualifiée. Mais Jacques Bacot se conforme-t-il uniment à une approche « rationaliste » de la fiction?

\title{
Vivre la fiction ? Une ouverture interculturelle
}

\author{
Soit par exemple à connaître les structures du \\ mythe. On sait comme ont été décevantes les \\ tentatives de mythologie. Elles l'auraient peut-être \\ été moins si nous avions appris à écouter le mythe \\ comme on écoute le récit d'un informateur sur le \\ terrain : c'est-à-dire le ton, l'allure, le rythme, les \\ récurrences, non moins que le contenu manifeste. \\ Vouloir comprendre le mythe comme une \\ proposition, par ce qu'il dit, c'est appliquer à une \\ langue étrangère notre grammaire, notre \\ vocabulaire.Maurice Merleau-Ponty (1960 : 159).
}

Pour poursuivre l'enquête, il convient en effet de manipuler avec circonspection la notion de fiction en différenciant ses ancrages philosophiques initiaux. En outre, il sera prudent de ne pas postuler a priori qu'aux anciennes représentations se substituent, comme mécaniquement de nouvelles «fables». Les modèles anciens ne font plus sens pour le voyageur du tournant $d u x x^{e}$ siècle ; cela, certes, témoigne d'un moment plus largement critique dans l'imaginaire social. Mais, précisément, comment s'opère le renouvellement de l'imaginaire ? Les représentations projetées sur le Tibet au XIX ${ }^{e}$ siècle étaient en bonne partie une conséquence de l'ignorance de l'Europe sur ce "pays inconnu ». La rencontre des explorateurs avec le Tibet et les Tibétains modifie-t-elle la donne? De quelle nature est leur découverte ? Comment accèdent-ils eux-mêmes à un surcroît de connaissance et conçoivent-ils de nouveaux modes de représentation? Enfin, l'opposition fiction/ connaissance n'est-elle pas dépassée dans pareils moments de transition épistémique? Autrement dit, au-delà de la posture réflexive qui caractérise la prise de distance de ce voyageur par rapport à ses prédécesseurs, c'est, plus positivement, la manière dont Bacot entre en contact avec le monde tibétain qui doit retenir notre attention.

Au-delà du repérage strict des phénomènes de circulation de motifs fictionnels ponctuels dans la littérature viatique, ce sont maintenant les questions des attitudes fictionnelles et de l'efficacité de la fiction dans la pratique du voyage même qui doivent retenir l'enquête poursuivie ici. À cet égard, Bacot, comme les autres, n'est-il pas parti pour le Tibet séduit par un imaginaire du voyage et un faisceau de représentations propres à l'air du temps? Si Caillié est parti pour Tombouctou, c'est qu'il avait d'abord lu Robinson Crusoé et rêvé 
sur la carte vierge du continent africain (Venayre 2002: 50-51). De même, Gabriel Bonvalot, le premier explorateur français à avoir traversé le Tibet ne se laissa-t-il pas aller, avant de partir, à une profonde rêverie sur les zones encore vierges de la carte de l'Asie (Bonvalot 1892: 1-6) ? Si l'on peut attester que son imaginaire de l'ailleurs était nourri des lectures de romans d'aventures (Ibid.: 1, 6, 186), on peut à tout le moins supputer qu'il avait également lu Jules Verne, et que, sans doute, Robur-le-Conquérant ou La Maison-à-vapeur lui étaient familiers. En évoquant des vers d'Othello, Fernand Grenard ne faisait-il pas référence à une bibliothèque du voyage ? Francis Younghusband (1910), explorateur et officier britannique, quant à lui, ne se rendait-il pas au Tibet comme Kim, le personnage de Kipling (2005 [1901]), avant lui (McMillin 2001)? Jacques Bacot (1925 : 122), de son côté, avoue être parti vers l'Asie avec en tête les décors de Lakmé ${ }^{17}$. De fait, on trouve des références à une littérature exotiste dans ses récits de voyage. Encore, Henri d'ollone (1911 : 292), officier français explorant à la même période que Bacot les confins sino-tibétains, ne manque pas d'évoquer les romans de Gustave Aymard et de Fenimore Cooper.

Il faut en convenir, l'exploration participe bien d'un imaginaire de l'aventure, lequel agit ici comme modèle de représentation de l'ailleurs autant que comme schème d'action. Mais jusqu'à quel point les explorateurs et, partant, tout voyageur, sont-ils condamnés à voyager comme des solitaires dans un rêve sans partage avec le monde qu'ils rencontrent?

Pareille approche soutient, peu ou prou, l'unicité des matrices culturelles et un cloisonnement indépassable des imaginaires (Bishop 1989; Buisine 1998), voire une violence symbolique exercée sur l'autre du voyage (Said 1979). Or, la lecture d'un récit comme celui de Bacot n'autorise pas, j'aimerais le montrer, la réduction du voyage à cet enfermement indépassable dans la culture propre du voyageur.

C'est en quelque sorte la véritable découverte de Jacques Bacot, laquelle n'est plus de l'ordre de l'exploration scientifique proprement dite, mais concerne pourtant encore la représentation de l'espace. Cette découverte est en lien étroit avec la présente discussion dans la mesure où elle se fait par le biais de récits oraux, d'histoires en circulation, à propos d'une région inconnue des Tibétains eux-mêmes, appelée "Népémakö», à la recherche de laquelle partent les habitants du Tibet sud-oriental alors mis à feu et à sang par le général chinois Zhao Erfeng. Or, Bacot part avec ces Tibétains. Ainsi le voyageur en vient-il à se représenter l'espace tibétain non plus uniquement en fonction de ses propres schèmes mais en accordant toute leur valeur de vérité aux représentations que lui permet d'acquérir son contact avec les Tibétains. Voici les modalités du récit de cette découverte :

« Népémakö est dans le Tibet et les Tibétains viennent seulement de le découvrir. Avant, c'était la Terre du Sud, demeure fabuleuse du monstre Shengui, "où les hommes ne pouvaient aller". On ne savait où elle était. Puis on apprit qu'il fallait d'ici aller vers le couchant, vers l'Inde brûlante, pendant une lune et demie, et traverser de nombreux fleuves. Des lamas très savants et très saints avaient reconnu la Terre promise d'où le travail et la mort seraient bannis, puisqu'il suffisait de cueillir les fruits de la terre et que, d'après les livres, on y jouirait de l'immortalité jusqu'au retour des temps meilleurs.

Voilà tout ce que savaient sur Népémakö les gens de ce village : des poèmes... et ils sont partis »(Bacot 1912 : 163-164).

Nous pouvons observer comment l'énonciation s'ouvre sur une certaine polyphonie : le présent de narration alterne avec du discours rapporté (les guillemets signalent une 
citation) et du discours indirect libre où la responsabilité de l'énoncé devient ambiguë ( " on... »). Observons encore les modalités et la dimension pragmatique de la transmission du savoir sur l'espace dont il est question ici; Bacot souligne son origine livresque et mythique, il souligne encore comment ces histoires qu'on se raconte et ces " poèmes " opèrent comme schèmes d'action. Il convient de rapporter l'usage de la fiction à une conception plus globale :

«Les Tibétains, c'est pour cela que je les aime, ne s'alarment pas des réalités. Les fictions seules les émeuvent; ils gardent pour elles seules leur sensibilité et des larmes. Ils sont à la fois stoïciens et poètes, hommes d'action et contemplateurs. Tout cela serait contradictoire chez un peuple civilisé et utilitaire. Mais de tout temps des âmes rêveuses ont habité le corps rugueux des nomades "(Ibid. : 92).

On peut ainsi observer la posture fondamentalement anti-rationaliste de l'explorateur. C'est cette posture particulière qui permet de rendre compte de l'infléchissement du cours du voyage qui se répercute dans le sous-titre du récit: Vers Népémakö, Terre promise des Tibétains. En effet, Bacot raconte, alors qu'il approche de Népémakö :

« En montant, j'ai vaguement conscience que je tombe dans l'inconnu en quittant le bassin de la Salouen. Que vais-je trouver? un plateau? le bassin de l'Irrawady? le bassin du Bramapoutre? je ne me le demande pas ; comme les Tibétains, je vis un peu dans un rêve. Sans critique, j'imagine déjà un horizon infini, des plaines, peutêtre des lacs, des villes jusqu'à l'Himalaya lointain, jusqu'à l'Inde »(Ibid. : 218-219).

Aussitôt prise la décision de prendre la direction de Népémakö, il formulait plus haut le mode selon lequel son voyage prenait un tour nouveau :

«Qu'importe si je vais à une déception, pourvu que l'illusion qui y mène soit belle. M'assurât-on que je verrai Népémakö et n'en reviendrai pas, je partirais encore. Et puis, sans espérer y atteindre, rien que suivre la trace de ces hommes qui sont partis, sur la foi de poèmes, vers leur Terre promise, n'est-ce pas un pèlerinage ?»( Ibid. : 165).

Son récit suggère ainsi que son équipée est désormais perçue comme un "mythe vécu ». La notion de "mythe vécu ", expulsée par l'anthropologie structurale, est reconsidérée aujourd'hui ${ }^{18}$. Deux décennies après le voyage de Bacot, Lucien Lévy-Bruhl et Maurice Leenhard $\mathrm{t}^{19} \mathrm{ont}$, les premiers, théorisé la notion. Lévy-Bruhl part d'une contestation du "principe de non-contradiction » en tant que seule procédure logique valide. Pour LévyBruhl, dans La Mythologie primitive (1935), les «mythes sont moins des histoires que l'on raconte qu'un milieu dans lequel les individus vivent, et dont les éléments sont considérés comme aussi réels que ceux donnés dans la perception » (cité in Keck 1993: 309).

Si la notion de «mythe vécu » permet de caractériser la manière dont Bacot perçoit la réponse des Tibétains à ce que, dans un premier temps, il qualifie de "fictions", il convient alors de s'interroger sur la nature de celles-ci. Loin d'y voir des affabulations fantaisistes, le voyageur les décline selon une "variation imaginaire». Formule développée plus tard par Paul Ricœur qui parle de « variation imaginative », elle doit être rapportée en premier lieu à la lecture de Husserl par Maurice Merleau-Ponty $(1960)^{20}$ :

« Nous ne pouvons dilater notre expérience des rapports sociaux et former l'idée des rapports sociaux vrais que par analogie ou par contraste avec ceux que nous avons vécus, bref par une variation imaginaire de ceux-ci, au regard de laquelle sans doute, ils recevront une signification neuve - comme la chute d'un corps sur un plan incliné est mise dans une nouvelle lumière par l'idée pure de la chute libre -, mais à laquelle ils fourniront tout ce qu'elle peut avoir de sens sociologique "(ibid. : 116). 
un processus étroitement comparable, Bacot évoque les «fables », les " poèmes » des Tibétains dans les termes qui correspondent à son univers fictionnel d'Européen : «terre promise» biblique, "odyssée » homérique (1912: 12), etc... Sans que soit impliquée a priori une archétypie transculturelle de la fiction et des symboles (Eliade 1952 : 229), ici s'établit un croisement - provisoire (le temps du voyage) et heuristique (assurant les modalités du vivre-ensemble de la petite communauté formée dans le voyage) - des imaginaires, culturellement distincts, du voyage et de l'espace. La particularité de ce croisement est d'opérer comme savoir paradoxal : paradoxal en tant qu'il est vécu, témoignant d'une sorte de "savoir » pratique, insu, dans l'instant du voyage (la capacité à vivre le mythe), et en tant qu'il débouche pourtant, grâce à des procédés d'analogie (les comparaisons interculturelles), sur un apport épistémique, et, nous le verrons, sur un renouvellement des champs de savoir en sciences humaines. En d'autres termes, et pour résumer, Bacot découvre in vivo une particularité culturelle incarnée dans les conduites des Tibétains, conduites du type de l'«immersion fictionnelle » (Schaeffer 1999 et 2005). Or, il est remarquable que la découverte de Bacot s'effectue par le biais de représentations symboliques et mentales auxquelles il accède luimême par immersion, transcendant ainsi un ensemble de différences d'ordre culturel, qui implique notamment pour le voyageur de se départir d'un certain « rationalisme ».

Pour rendre compte du faisceau complexe des processus fondamentaux à l'œuvre, il me paraît fécond de rapporter la notion de métalepse ontologique (Schaeffer 2004) ${ }^{21}$ aux actualisations de la fiction dégagées jusqu'ici. La métalepse, considérée comme «renégociation permanente entre immersion et attention distancée accordée au mimème » (Ibid. : 333), permettrait de rendre compte de la "co-événementialité de deux actes communicationnels logiquement incompatibles qui sont émis par un même sujet ", un « sujet bicéphale » (Ibid. : 328). Ainsi la «métalepse cognitive » que décrit Schaeffer se rapproche-t-elle du savoir paradoxal à l'œuvre dans le croisement des imaginaires culturels évoqués précédemment, en cela que cette acception de la métalepse relève non seulement d'une "technique narrative» mais également d'une capacité de partage expérientiel sans que soit pour autant invoquée une « hallucination » ontologique ou une irrationalité logique (Ibid. : 333). Transposée au récit de voyage, la métalepse me semble permettre de penser les "frottements " logiques qui échappent précisément à une approche rationaliste tout en autorisant le bénéfice épistémique du " heurt » cognitif (Bonoli 2008) ou de la «défamiliarisation» culturelle (Ginzburg 2001) qu'implique une telle « immersion ».

Concrètement, le «mimème » prend ici des formes labiles et non matérielles : mythe, récits oraux. Or, « le mimème ne prend pas la place de l'environnement réel » et « l'état clivé de l'immersion fictionnelle s'explique en termes d'interaction entre différents niveaux de traitement mental » (Schaeffer 2004 : 332). Aussi, la métalepse « aménage une voie de passage entre les deux univers tout en maintenant leur co-fonctionnement » (Ibid . :331). On saisit dès lors le bénéfice que représente la notion de métalepse pour penser la situation interculturelle vécue par le voyageur. Si, comme l'affirme Jean-Marie Schaeffer, l'«immersion mimétique » fonctionne selon une «dynamique tensionnelle dans laquelle des moments de simulation mimétique et des moments métaleptiques ne cessent d'alterner ", la caractéristique du récit de Bacot est de signaler un fonctionnement cognitif valable pour le voyageur, lequel «pratique » les œuvres de fictions littéraires (détermination culturelle) et élabore dans le même moment un réseau de représentations et de correspondances, notamment intertextuelles. Celles-ci deviennent alors 
correspondances interculturelles : le bénéfice cognitif vaut pour lui, et pour son lecteur. Mais le récit veut, en outre, décrire une modalité de connaissance qui serait propre aux Tibétains : la métalepse agit dès lors comme outil cognitif et heuristique. En définitive, la métalepse permet de décrire une procédure «intuitive» de la connaissance où interviennent des «niveaux différents de traitement mental » pour reprendre les termes de Schaeffer (Ibid. : 333-334).

À ces divers titres, les processus fictionnels mis au jour dans le récit de Bacot ne sont en aucun cas le signe d'une intransitivité culturelle ou épistémologique; ils débouchent au contraire sur un apport de connaissances neuves. En effet, l'expérience viatique particulière à Bacot engendre deux orientations nouvelles dans l'histoire des représentations et des savoirs sur le Tibet. D'une part, sur un plan épistémologique, loin de la conception géologique de l'exploration que nous avons observée précédemment, l'attention de Bacot se déplace sur les représentations, celles de sa culture propre et celles des Tibétains. D'autre part, la découverte de Népémakö était proprement inattendue avant le voyage. Elle est perçue comme un «mythe vécu » dans le moment du voyage mais implique un nouveau champ de questionnement pour les études tibétaines. À la suite de Bacot, l'orientalisme français portera son attention sur la tradition tibétaine des gter ma. Ce sont, littéralement, des "trésors» de diverses natures, cachés par Padmasambhava, maître venu de l'Inde qui introduisit le bouddhisme au Tibet au viII siècle. Parmi ces «trésors", sources d'un véritable "genre littéraire " assimilable à des guides de pèlerinage (Buffetrille $2007:$ 3), figurent en particulier les sbas yul, « "pays” [...] "cachés" pour qu'il[s] soi[en]t "découvert[s]" à une époque donnée annoncée dans des prophéties, par un être prédestiné, un "découvreur de trésor" " (Ibid.: 2). Népémakö en est, en 1909, l'une des plus récentes manifestations ${ }^{22}$.

Ainsi apparaît un changement fondamental dans les milieux de l'orientalisme: avec Bacot, on assiste à la naissance de la tibétologie française moderne et d'une réflexion centrée sur le Sacré et les « formes symboliques » tibétaines, grâce à la découverte d'une tradition «littéraire " et «imaginaire » particulière. De par son hétérogénéité épistémologique, l'expérience racontée par Bacot s'inscrit dans un faisceau d'implications double qui indique un champ de questionnements en partage avec deux formes de savoir : je veux parler, d'une part, de l'impact du récit de Bacot sur le développement et la modification des questionnaires de la science; et, d'autre part, de l'impact du récit de Bacot sur les représentations littéraires. En effet, Victor Segalen était un lecteur enthousiaste du Tibet révolté de Bacot et sa présentation du Tibet, dans Équipée (1916) et Thibet (1919), mais également dans ses notes de voyage, Briques et Tuiles (1915) et Feuilles de route (1916), portent la marque de cette lecture. Les relations entre fiction et récit de voyage sont alors redistribuées selon un ordre nouveau dans ces œuvres dont l'ambition de renouveler les catégories génériques littéraires est clairement affichée ${ }^{23}$. L'« œuvre tibétaine » de Segalen peut alors être lue elle-même comme une "variation imaginaire » du Tibet révolté par Bacot. Variations imaginaires, Équipée et Thibet de Segalen prolongent ce qui est déjà une variation imaginaire, d'ordre ethnographique, mais relevant d'une anthropologie au sens large, où sont exhibées les dimensions connexes de la fiction et du mythe vécu. 


\title{
Récit de voyage et fiction : trois ordres de décrochements symboliques
}

\author{
Dans ces centaines de rencontres quotidiennes \\ entre l'Imaginaire et le Réel, j'ai été moins \\ retentissant à l'un d'entre eux, qu'attentif à leur \\ opposition. - J'avais à me prononcer entre le \\ marteau et la cloche. J'avoue, maintenant, avoir \\ surtout recueilli le son.Victor Segalen, Équipée
}

(1995 : II, 136).

En dernière analyse, j'aimerais proposer que les relations entre expérience et fiction dans le récit de voyage s'organisent selon trois niveaux ou ordres de décrochements symboliques auxquels s'appliquerait la notion de métalepse.

À un niveau épistémologique, d'abord, en ce qui concerne la régulation des formes de savoir en contexte interculturel, la métalepse permet de penser conjointement, d'une part un ensemble de représentations partagées propres à un groupe socioculturel précis au sein duquel l'on distingue d'abord représentations fictionnelles (fiction littéraire; imaginaire du voyage, mystique de l'aventure) puis représentions scientifiques, et, d'autre part, un ensemble de pratiques où l'on distingue pratiques littéraires et pratiques scientifiques (l'exploration, la géographie, l'anthropologie), ensembles engageant des procédures de connaissance propres à ce même groupe ; ce à quoi j'ai limité ici l'usage du terme "défamiliarisation " (incarnée dans les rapports entre fiction littéraire et texte non fictionnel). L'acception du terme "fiction" ici serait l'acception restreinte de "production symbolique artistique ».

À un niveau référentiel, ensuite, en ce qui concerne le processus d'ajustement des savoirs à l'expérience vécue, la métalepse permet de penser les relations de ces ensembles de représentations et de pratiques avec un " ailleurs », une expérience du monde (le paysage « tibétain »). C'est ce à quoi j'ai limité l'usage de la notion de « heurt» (décrire le monde inconnu dans les termes de modèles dont il s'agit de discuter l'adéquation). L'acception du terme «fiction» prend ici une dimension nettement constructiviste et rejoint la définition moins d'une «représentation» que d'une "présentation» (Bonoli 2008: 61-67).

42 Enfin, à un niveau culturel et anthropologique - et c'est la particularité supplémentaire de mon dernier exemple où est discutée la commensurabilité possible de systèmes culturels différents -, la notion de métalepse permet de penser la rencontre entre des «formes symboliques » hétérogènes, culturellement disparates, et de rendre compte d'un bénéfice épistémique débordant l'immersion participative proprement dite. Ici, le récit de voyage fait état des modalités représentationnelles et actionnelles tibétaines par le biais d'une expérience partagée. La "fiction» est alors envisagée comme une simulation partagée par une communauté d'individus d'appartenances culturelles variées.

Par ce rapide panorama des manifestations de la fiction dans les récits français d'exploration du Tibet, concentrés sur une période relativement restreinte, j'ai pu signaler différents phénomènes de transferts transgénériques et de processus à la fois historiques (nous parlerions alors d'une culture française de l'exploration du Tibet) et cognitifs (la fiction, dans ce corpus restreint, prend sens, on l'a vu, selon des traditions 
épistémologiques antagonistes) qu'il convient de comprendre dans la durée. J'ai été amené à parler de différents décrochements symboliques occasionnés par la présence de dimensions fictionnelles variables dans le récit de voyage: heurt, défamiliarisation, évolution des représentations, mythe vécu, savoir paradoxal, schème d'action, variation imaginaire, métalepse ontologique. Autant de notions applicables aux rapports, remarquablement polymorphes, entre fiction et récit de voyage et rendant compte de la diversité des usages et des acceptions du terme "fiction ». Ce faisant, une alternative se dessine aux options théoriques qui ont divisé la critique sur la question, dans la mesure où elles permettent de rendre compte des différentes manifestations fictionnelles sans perdre de vue la question de l'expérience au fondement même d'une définition du récit de voyage.

En retour, le récit de voyage alimente durablement la littérature dite fictionnelle. De l'hétérogénéité du discours romanesque chez Jules Verne, à la disqualification et réinvention des formes génériques chez Victor Segalen, il semble ainsi que le récit de voyage nourrisse de manière discontinue mais durable la réflexion littéraire sur les rapports entre monde et fiction, réflexion inscrite dans des paradigmes dont on ne peut ignorer l'évolution à travers le temps.

Ainsi, s'il faut reconnaître les ressemblances globales entre les différentes manifestations de la « fiction » inventoriées, il n'apparaît pas moins nécessaire, en dernier examen, de définir au cas par cas la notion et de décrire l'usage local qui en est fait, afin de préciser les enjeux esthétiques et cognitifs sur lesquels elle ouvre. Interdisant autant de renvoyer dos à dos fiction et récit de voyage que de verrouiller dans une théorie unifiée la question de leurs rapports, l'étude de ces derniers se place du même coup dans la perspective double d'une historicisation et d'une anthropologie du fait littéraire dont relève pleinement la littérature viatique.

\section{BIBLIOGRAPHIE}

Antoine, Philippe 1997 Les Récits de voyage de Chateaubriand. Contribution à l'étude d'un genre. Paris, Honoré Champion (« Romantisme et Modernité »).

Antoine, Philippe \& Marie-Christine Gomez-Géraud, eds 2001 Roman et récit de voyage. Écriture de fiction, écriture du voyage. Actes du colloque de l'Université de Picardie-Jules Verne, décembre 1999. Paris, Presses de l’Université Paris-Sorbonne (« Imago Mundi »).

Bacot, Jacques 1909 Dans les Marches tibétaines. Autour du Dokerla, novembre 1906-janvier 1908. Paris, Plon-Nourrit.

1912 Le Tibet révolté. Vers Népémako, la Terre promise des Tibétains. Paris, Hachette [rééd. : Paris, Raymond Chabaud, 1988].

1925 «Ce qui sépare encore l'Orient et l'Occident », Les Cahiers du mois 9-10 : Les Appels de l'Orient : 110-123.

Berdoulay, Vincent 1995 [1981] La Formation de l'École française de géographie (1870-1914). Paris, Éd. du cths. 
Bessière, Jean 2004 « Récit de fiction, transition discursive, présentation actuelle du passé, ou que le récit de fiction est toujours métaleptique », in John Pier \& Jean-Marie Schaeffer, eds, Métalepses... : 279-294.

Bishop, Peter 1989 The Myth of Shangri-La. Tibet, Travel Writing and the Western Creation of Sacred Landscape. London, Athlone Press.

Bonoli, Lorenzo 2008 Lire les cultures. La connaissance de l'altérité culturelle à travers les textes. Paris, Kimé.

Bonvalot, Gabriel 1892 De Paris au Tonkin à travers le Tibet inconnu. Paris, Hachette.

Buffetrille, Katia 2000 Pèlerins, lamas et visionnaires. Sources orales et écrites sur les pèlerinages tibétains. Wien, Arbeitskreis fur Tibetische und Buddhistische Studien (« Wiener Studien zur Tibetologie und Buddhismuskunde » 46).

2007 «"Pays caché” ou "Avenir radieux ?" : Le choix de Shes rab rgya mtsho », in Birgit Kellner et al., eds, Pramānakirtih. Papers dedicated to Ernst Steinkellner on the Occasion of his 70th Birthday. Wien, Arbeitskreis fur Tibetische und Buddhistische Studien (« Arbeitskreis fur Tibetische und Buddhistische Studien » 70) : 1-22.

Buisine, Alain 1998 L'Orient voilé. Paris, Zulma.

Deleuze, Gilles 1993 Critique et Clinique. Paris, Minuit.

Didier, Hugues 1996 Les Portugais au Tibet. Les premières relations jésuites (1624-1635). Paris, Chandaigne.

Dodin, Thierry \& Heinz Räther, eds 2001 Imagining Tibet. Perceptions, Projections, and Fantasies. Boston, Wisdom.

Dufief, Anne-Simone, ed. 2010 Louis, Victor et Théodore: les Pavie, une famille angevine au temps du romantisme. Angers, Presses universitaires d'Angers.

Duncan, James \& Derek Gregory, eds 1999 Writes of Passage. Reading Travel Writing. London-New York, Routledge.

Dutreuil de Rhins, Jules-Léon 1897-1898 Mission scientifique dans la Haute Asie, 1890-1895. Éd. par Fernand Grenard. Paris, E. Leroux. 3 vol.

Eliade, Mircea 1952 Images et Symboles. Essai sur le symbolisme magico-religieux. Paris, Gallimard.

Flahaut, François \& Nathalie Heinich, eds 2005 L'Homme 175-176 : Vérités de la fiction. Paris, Éd. de l'EHEss.

Genette, Gérard 2004 « De la figure à la fiction », in John Pier \& Jean-Marie Schaeffer, eds, Métalepses... : 21-36.

Ginzburg, Carlo 1989 « Traces : racines d'un paradigme indiciaire ", in Mythes, emblèmes, traces. Morphologie et histoire. Paris, Flammarion : 139-180.

2001 «L'estrangement : préhistoire d'un procédé littéraire », in A distance. Neuf essais sur le point de vue en histoire. Paris, Gallimard: 15-36.

Gomez-Géraud, Marie-Christine, ed. 1990 Les Modèles du récit de voyage. Nanterre, Centre de recherche du département de français de Paris 10 - Nanterre («Littérales 7 ).

Gournay, Dominique 2004 Pour une poétique de Thibet de Victor Segalen. Besançon, Presses universitaires franc-comtoises.

Grenard, Fernand 1904 Le Tibet. Le pays et les habitants. Paris, Armand Colin. 
Guentner, Wendelin 1997 Esquisses littéraires. Rhétorique du spontané et récit de voyage au XIXe siècle. St-Genouph, Nizet.

Hilton, James 1933 Lost Horizon. London, Macmillian [rééd. : New York, Pocket Books, 1995].

Jacob, Christian \& Frank Lestringant, eds 1981 Arts et légendes d'espaces. Figures du voyage et rhétoriques du monde. Paris, Presses de l'École normale supérieure.

Jamin, Jean 2005 « Fictions haut régime : du théâtre vécu au mythe romanesque », l'Homme 175-176 : Vérités de la fiction : 165-202.

Keck, Frédéric 1993 Le Problème de la mentalité primitive. Lévy-Bruhl, entre philosophie et anthropologie. Lille, Université Charles-de-Gaulle-Lille 3, thèse de doctorat.

2007 « Mentalité mythique ou mystique ? Leenhardt et Lévy-Bruhl », in Michel Naepels \& Christine Salomon, eds, Terrains \& destins de Maurice Leenhardt.Paris, Éd. de l'EHEss: 51-67. 2008 Contradiction et participation. Lucien Lévy-Bruhl, entre philosophie et anthropologie. Paris, Éd. du CNRS.

Kipling, Rudyard 2005 [1901] Kim. Éd. par Alexis Tadié. Paris, Gallimard (« Folio. Classique »).

Laboulais-Lesage, Isabelle, ed. 2004 Combler les blancs de la carte. Modalités et enjeux de la construction des savoirs géographiques ( $\mathrm{XVI}^{e}-\mathrm{XX}{ }^{e}$ siècle). Strasbourg, Presses universitaires de Strasbourg («Sciences de l'histoire »).

Large-Blondeau, Anne-Marie 1960 « Les pèlerinages tibétains », in Les Pèlerinages. Paris, Le Seuil (« Sources Orientales » 3 ) : 199-246.

Lejeune, Dominique 2007 [1991] La France de la Belle Époque, 1896-1914. Paris, Armand Colin.

Lopez, Donald S. Jr. 1998 Prisoners of Shangri-La. Tibetan Buddhism and the West. Chicago, Chicago University Press [trad. franç. : Fascination tibétaine, Paris, Autrement, 2003].

McMillin, Laurie Hovell 2001 English in Tibet, Tibet in English. Self-Presentation in Tibet and the Diaspora. New York, Palgrave MacMillan.

Meizoz, Jérôme 2004 « "Postures" d'auteur et poétique (Ajar, Rousseau, Céline, Houellebecq) », Vox poetica [http ://www.voxpoetica.org/t/meizoz.html].

Merleau-Ponty, Maurice 1960 Éloge de la philosophie. Paris, Gallimard (« Tel »).

Montalbetti, Christine 1997 Le Monde, le voyage et la bibliothèque. Paris, Presses universitaires de France («Écritures »).

Moureau, François, ed. 1986 Métamorphoses du récit de voyage. Actes du colloque de la Sorbonne et du Sénat (2 mars 1985). Paris-Genève, Champion-Slatkine.

Moussa, Sarga 1995 La Relation orientale. Enquête sur la communication dans les récits de voyage en Orient (1811-1861). Paris, Klincksieck (« Littérature des voyages »).

Ollone, Henri d' 1911 Les Derniers Barbares, Chine-Tibet-Mongolie (Mission d'Ollone 1906-1909). Paris, Pierre Lafitte.

Pasquali, Adrien 1994 Le Tour des horizons. Critique et récit de voyage. Paris, Klincksieck.

Pier, John \& Jean-Marie Schaeffer, eds 2004 Métalepses. Entorses au pacte de la représentation. Paris, Éd. de l'EHESs (« Recherches d'histoire et de sciences sociales » 108).

Rabau, Sophie 2002 L'Intertextualité. Paris, GF Flammarion («Corpus »). 
Reclus, Élisée 1882a Nouvelle Géographie universelle. La Terre et les hommes, 7. L'Asie orientale. Paris, Hachette.

$1882 \mathrm{~b}$ Histoire d'une montagne. Paris, Hetzel.

Reichler, Claude 2002 « Littérature et anthropologie : de la réprésentation à l'interaction dans une Relation de la Nouvelle-France au XVII ${ }^{\mathrm{e}}$ siècle ", L'Homme 164 : 37-56.

2005 « Pourquoi les pigeons voyagent : remarques sur les fonctions du récit de voyage », Versants $50: 11-36$.

Roudaut, Jean 1984 «Quelques variables du récit de voyage ", Nouvelle Revue française 377 : 58-70.

Said, Edward 1979 Orientalism. London, Routledge.

Schaeffer, Jean-Marie 1999 Pourquoi la fiction ? Paris, Le Seuil.

2004 « Métalepse et immersion fictionnelle », in John Pier \& Jean-Marie Schaeffer, eds, Métalepses ...: 323-334

2005 «Quelles vérités pour quelles fictions? », L’Homme 175-176 : 19-36.

Schaffner, Alain 2001 « "Ce n'est pas un livre que j'écris." Équipée de Victor Segalen : le récit de voyage en question ", in Philippe Antoine \& Marie-Christine Gomez-Géraud, eds, Roman et récit de voyage... : 81-90.

Segalen,Victor 1995 Euvres complètes. Éd. par Henry Bouillier. Paris, Robert Laffont («Bouquins »). 2 vol.

Tadié, Alexis 2006a « D'Othello à Otello : les avatars du Maure », Études Épistémè 9 : 409-427. 2006b « Relire Othello : un mythe nomade », Revue Silène [http://www.revuesilene.com/f/ index.php?sp=liv\&livre_id=53].

Thévoz, Samuel 2007 « Paysage et nomadismes dans Le Tibet révolté de Jacques Bacot », A contrario. Revue de sciences sociales et humaines 1 (5) : 8-23.

2011 « Victor Segalen et les explorateurs du Tibet : le "pays au-delà" et la crise du paysage ", Revue d'histoire littéraire de la France 4 : 901-930.

Venayre, Sylvain 2002 La Gloire de l'aventure. Genèse d'une mystique moderne 1850-1940. Paris, Aubier.

Venayre, Sylvain, ed. 2006 Sociétés \& Représentations 21 : Le Siècle du voyage. Paris, Publications de la Sorbonne.

Verne, Jules 1880 La Maison-à-vapeur. Paris, Hetzel (« Bibliothèque d'éducation et de récréation »).

2004 [1886] Robur-le-Conquérant. Paris, Le Livre de Poche.

Weber, Anne-Gaëlle 2006 « Le genre romanesque du récit de voyage scientifique au XIX siècle ", in Sylvain Venayre, ed., Sociétés \& Représentations 21... : 59-77.

Younghusband, Francis 1910 India and Tibet, 1903-1904. London, John Murray.

Zufferey, Joël 2006 Le Discours fictionnel. Autour des nouvelles de Jean-Pierre Camus. Louvain, Peeters (« La République des lettres »).

\section{NOTES}

1. Voir Adrien Pasquali (1994) pour une recension des approches multiples du récit de voyage. L'auteur évoque notamment l'hétérogénéité discursive du genre comme 
expliquant la diversité des regards portés sur le récit de voyage. Depuis lors, la littérature critique s'est considérablement développée; on comprendra que le présent article ne cherche guère à épuiser la question par un inventaire exhaustif, mais propose une synthèse des positions critiques sur la relation entre récit de voyage et fiction.

2. Sous des angles divers, l'approche a suscité de nombreuses publications : entre autres, dans la présente bibliographie, Antoine \& Gomez-Géraud (2001), Duncan \& Gregory (1999), Gomez-Géraud (1990), Guentner (1997), Jacob \& Lestringant (1981), Moureau (1986).

3. À titre d'exemple, mentionnons ici les voyageurs romantiques en Orient (Moussa 1995) ou les dimensions intertextuelles et autoréférentielles dans les récits de Chateaubriand (Antoine 1997).

4. C'est la thèse platonicienne "antimimétique » ou "rationaliste ", selon Jean-Marie Schaeffer (1999). Pour une synthèse critique des différentes théories de la fiction, cf. Joël Zufferey (2006).

5. Claude Reichler a ainsi défini a minima le récit de voyage selon quatre "instances »: «1) la narration (ou relation) 2) d'un déplacement effectué 3) par le narrateur voyageur 4) et adressée à un lecteur ». Celles-ci, complète l'auteur, sont "nécessaires pour accomplir les fonctions du récit de voyage: testimoniale, épistémique, esthétique » (2005: 13).

6. Soulignons également la dimension énonciative particulière du passage : qui parle ? Il y a une forte ambiguïté entre le détenteur du savoir sur le monde qu'est le narrateur extradiégétique et le regardeur-jouisseur qu'est le personnage.

7. Celles, notamment, du jésuite portugais Antonio de Andrade qui évoquait, en 1626, le «formidable désert » tibétain dans les termes d'un locus horridus : « Il n'y a pas de village, car il n'y a pas de lieu où il puisse y en avoir, pas un arbre, pas une herbe, et rien d'autres si ce n'est des rocailles recouvertes par la neige qui y tombe continuellement ». Mais il convient de noter que la description est, ailleurs, nettement plus nuancée; il n'en demeure pas moins que ce pan négatif se déposera pour longtemps de manière univoque dans la mémoire collective, cf. Hugues Didier (1996 : 41).

8. Cf., à propos de l'auteur, Anne-Simone Dufief (2010). Sur la dimension topique de la description du Tibet, de nombreuses études ont été produites pour le domaine anglosaxon, cf. en particulier Peter Bishop (1989), Thierry Dodin \& Heinz Räther (2001) et Donald Lopez (1998).

9. Pour l'anecdote, rappelons que Jules Verne affirmait à Hetzel avoir «fait la chasse à tout ce qui était altitudes, latitudes, longitudes ", cf. la lettre du 18 juin 1885 (in 2004 [1886] : 265).

10. À cette période, précisément, le voyage au Tibet relève exclusivement de l'exploration, participant par là même pleinement de la logique scientifique de la découverte thématisée par Jules Verne et accentuant du même coup la question de la connaissance dans le récit.

11. Celui-ci présente la particularité d'être la version abrégée du rapport de la Mission scientifique Dutreuil de Rhins en Haute Asie (1890-1895), dont Grenard a été le scripteur tant pour le récit de voyage que pour les résultats scientifiques.

12. L'imaginaire occidental du Tibet accordera une fonction symbolique et utopiste à ce motif de la « pyramide de cristal ». Voir le roman de James Hilton (1933). 
13. De toute évidence, sous la Troisième République, les explorateurs disposent, autant que d'une formation scientifique, de géographie principalement, d'une culture littéraire (Berdoulay 1995 : 77-108; Lejeune 2007 : 127-150). De fait, il est raisonnable de soutenir que le topos vernien du paysage tibétain, séquence d'un roman parmi toutes les autres publications de l'auteur dans la "Bibliothèque d'éducation et de récréation » de l'éditeur Hetzel, se retrouve comme naturellement dans les récits de voyage au Tibet de type scientifique. Le même Hetzel publie notamment les ouvrages du géographe Élisée Reclus comme son Histoire d'une montagne (1882b). Entre 1876 et 1894, c'est Hachette, autre maison éditant des ouvrages à vocation de vulgarisation des savoirs, qui publie la Nouvelle Géographie universelle de Reclus; Verne, lecteur assidu de Reclus, a vraisemblablement emprunté thèmes et motifs à la section « Tibet » (Reclus 1882a).

14. Cf. Alexis Tadié (2006a et b) sur la fortune intertextuelle d'Othello en tant que «mythe nomade ».

15. Cf. Christine Montalbetti (1997). La poéticienne énumère avec finesse les disparités entre le monde et la langue que les voyageurs (romantiques) s'ingénient à gloser.

16. De "nature schisteuse ", l'Arka tâgh appartient à l'ère jurassique et est de formation assez récente par rapport au vieux continent auquel appartient l'Oustoun tâgh traversé précédemment. La citation d'Othello délimite ainsi un amont et un aval textuels qui se veulent le report de la rupture d'ordre géologique observée in situ.

17. Notons que l'exotisme est ici signalé comme une « déception juvénile ». Lakmé est un opéra de Léo Delibes (1883), dont le livret s'inspire de Rarahu de Pierre Loti (1880). Sur Loti et les fantasmes exotistes projetés sur le monde oriental, cf. Alain Buisine (1998).

18. Voir, dans cette perspective, Jean Jamin (2005) qui développe d'un point de vue anthropologique une réflexion fort stimulante sur les rapprochements possibles entre mythe, croyances, fiction. Voir également à ce propos Frédéric Keck (1993: 8-12 et 308-310).

19. Maurice Leenhardt exerce comme missionnaire en Nouvelle-Calédonie à partir de 1902 ; il est donc contemporain de Bacot.

20. Je dois encore à Jean Jamin (2005) l'usage de la notion dans ses « ressemblances de famille» avec le "mythe vécu», les croyances et la fiction. Merleau-Ponty noue sa réflexion à la réaction dont Husserl fit part à Lévy-Bruhl après la lecture de La Mythologie primitive. Dans «Le philosophe et la sociologie ", Merleau-Ponty reconsidère notamment la notion de monde primitif par rapport au monde historique. La division théorique, qui a tant fait couler d'encre, est celle que fait Lévy-Bruhl. L'auteur ne lui attribuait toutefois pas la valeur phylogénétique dont on l'a accusé dans le procès de son évolutionnisme (Keck 2008 : 25-58).

21. La notion de métalepse est bien entendu empruntée à la rhétorique classique, notamment par Genette, qui la confine à un fonctionnement narratologique (commutation accidentelle des niveaux énonciatifs). Cf. Gérard Genette (2004), et la contestation de cette réduction par Jean Bessière (2004), qui lui restitue sa valeur temporelle.

22. Cf. en particulier Anne-Marie Large-Blondeau (1960) et Katia Buffetrille (2000 et 2007).

23. Cf., pour Équipée, Alain Schaffner (2001) et, pour Thibet, Dominique Gournay (2004). Voir également Samuel Thévoz (2011) pour une étude de l'impact de la lecture des explorateurs français au Tibet sur l'œuvre de Segalen. 


\section{RÉSUMÉS}

\section{Résumé}

Samuel Thévoz, Des poèmes... et ils sont partis. La fiction dans les récits d'exploration au TibetDepuis les années 1980, la littérature de voyage a retenu l'intérêt des historiens et des littératures. Mais quels regards ont-ils portés sur le statut générique de ces textes? Plus précisément, comment ont-ils envisagé les relations entre récit de voyage et fiction? Des différentes approches proposées, que l'on comprendra ici dans une évolution des paradigmes en sciences humaines, on retiendra la méfiance générale sur le genre viatique et le monolithisme théorique, témoins d'une définition insuffisante du récit de voyage. Le présent article, faisant appel aux récents débats autour du statut ontologique et anthropologique de la fiction, cherche à distinguer, sur la base d'un corpus de récits de voyage circonstancié (le voyage français au Tibet au tournant $\mathrm{du} \mathrm{xx}^{\mathrm{e}}$ siècle), la diversité des phénomènes fictionnels au sein du récit de voyage, au loin d'une conception figée de leurs relations. Si les définitions « locales » et les usages multiples de la fiction plaident pour un traitement au cas par cas, il ressort également de l'analyse que, dans le contexte propre à la situation interculturelle que présente le récit de voyage, la fiction demande à être envisagée dans ses vertus heuristiques.

\section{Abstract}

Samuel Thévoz, "Poems, and They Went Away" : Fiction in Accounts of Travels in Tibet - Since the 1980s, travel literature has attracted the interest of historians and students of literature. What view have they adopted of the generic status of such texts? How have they conceived of the relation between travel accounts and fiction? In the different approaches proposed (understood herein as a shift in the paradigms of human sciences), we notice a general mistrust of travel narratives and a "theoretical monolithism ", this being evidence of an inadequate definition of the genre. A reference to recent debates about fiction's ontological and anthropological status serves to discern the diversity of fictional phenomena in a corpus of detailed French accounts of travels in Tibet at the turn of the $20^{\text {th }}$ century. The "local» definitions and multiple uses of fiction call for a case-by-case approach. In the intercultural situation presented in travel accounts, fiction should be seen in terms of its heuristic value.

\section{INDEX}

Mots-clés : littérature de voyage, fiction - Tibet - interculturalité, Jacques Bacot - Jules Verne Fernand Grenard

Keywords : Travel Writing, Interculturality

\section{AUTEUR}

\section{SAMUEL THÉVOZ}

Université de Lausanne Section de français, Dorigny (Suisse) 